\title{
SIMO-OFDM Channel Estimation based on Nonlinear Complex LS-SVM
}

\author{
Anis Charrada \\ Ph.D Student \\ Tunisia Polytechnic School, \\ Carthage University, Tunisia
}

\author{
Abdelaziz Samet \\ Associate Professor \\ Tunisia Polytechnic School, \\ Carthage University, Tunisia
}

\begin{abstract}
In this contribution, we propose a robust highly selective nonlinear channel estimator for Single -Input Multiple-Output (SIMO) Orthogonal Frequency Division Multiplexing (OFDM) system using complex Least Squares Support Vector Machines (LS-SVM) and applied to Long Term Evolution (LTE) downlink under high mobility conditions .The new method uses the information provided by the pilot signals to estimate the total frequency response of the channel in two phases: learning phase and estimation phase. The estimation algorithm makes use of the reference signals to estimate the total frequency response of the highly selective multipath channel in the presence of non-Gaussian impulse noise interfering with pilot signals. Thus, the algorithm maps trained data into a high dimensional feature space and uses the Structural Risk Minimization (SRM) principle to carry out the regression estimation for the frequency response function of the highly selective channel. The simulations show the effectiveness of the proposed method which has good performance and high precision to track the variations of the fading channels compared to the conventional LS method and it is robust at high speed mobility.
\end{abstract}

\section{Keywords}

LS-SVM, SIMO, MRC, impulse noise, OFDM, LTE.

\section{INTRODUCTION}

The major problem that wireless communication systems suffer is multipath fading caused by scattering of the transmitted signal. However, we can treat multipath propagation as multiple channels between the transmitter and receiver to improve the signal to noise ratio. While using Single-Input Multiple-Output (SIMO) systems, the diversity receivers extract multiple signal branches of the same signal received from different channels and apply gain combining schemes such as Maximum Ratio Combining (MRC) which gives better performance than Equal Gain Combining (EGC) or Selective Combining (SC).

The combination of OFDM and SIMO techniques in the same system increases spectral efficiency and improves link reliability without additional transmit power or bandwidth. By implementing the OFDMA access technique in LTE Downlink system, new approaches for time and frequency synchronization, equalisation and channel estimation are needed. In our knowledge, the use of Support Vector Machines (SVM) has been deployed in OFDM system and has not been explored in SIMO-OFDM systems.

Here, a proposed SVM robust version for nonlinear channel estimation in SIMO systems with the presence of nonGaussian impulse noise that is specifically adapted to pilotaided OFDM structure is presented. In fact, impulses of short duration are unpredictable and contain spectral components on all subchannels which impact the decision of the transmitted symbols on all subcarriers.

The channel estimation algorithm is based on the nonlinear complex least squares support vector machines (LS-SVM) method in order to improve communication efficiency and quality of OFDM systems. The principle of the proposed nonlinear LS-SVM algorithm is to exploit the information provided by the reference signal to estimate the channel frequency response. In highly selective multipath fading channel, where complicated nonlinearities can be present, the estimation precision can be lowed by using linear methods. So, we adapt the nonlinear LS-SVM algorithm which transforms the nonlinear estimation in low dimensional space into the linear estimation in high dimensional space, so it improves the estimation precision.

In this contribution, the proposed nonlinear complex LS-SVM technique is applied to LTE downlink highly selective channel using pilot symbols. For the purpose of comparison with conventional LS algorithm, we develop the nonlinear LSSVM algorithm in terms of the RBF kernel. Simulation section illustrates the advantage of this algorithm over LS algorithm in high mobility environment. The nonlinear complex LS-SVM method shows good results under high mobility conditions due to its improved generalization ability.

The scheme of the paper is as follows. Section 2 briefly introduces the OFDM system model. Then, multipath channel model is presented in section 3. Section 4 describes the SIMO-OFDM system. We develop the formulation of the proposed nonlinear complex LS-SVM channel estimation method in section 5. Section 6 presents the simulation results in SISO-OFDM and SIMO-OFDM cases respectively. Finally, in section 7 , conclusions are drawn.

\section{OFDM SYSTEM MODEL}

The OFDM system model consists firstly of mapping binary data streams into complex symbols by means of QAM modulation. Then data are transmitted in frames by means of serial-to-parallel conversion. Some pilot symbols are inserted into each data frame which is modulated to subcarriers through IDFT. These pilot symbols are inserted for channel estimation purposes. The IDFT is used to transform the data sequence $X(k)$ into time domain signal as follow:

$$
\begin{array}{r}
x(n)=I D F T_{N}\{X(k)\} \\
=\sum_{k=0}^{N-1} X(k) e^{j \frac{2 \pi}{N} k n}, \\
=0, \cdots, N-1
\end{array}
$$

One guard interval is inserted between every two OFDM symbols in order to eliminate inter-symbol interference (ISI). This guard time includes the cyclically extended part of the 
OFDM symbol in order to preserve orthogonality and eliminate inter-carrier interference (ICI). It is well known that if the channel impulse response has a maximum of $L$ resolvable paths, then the GI must be at least equal to $L$ [1].

Thus, for the OFDM system comprising $N$ subcarriers which occupy a bandwidth $B$, each OFDM symbol is transmitted in time $T$ and includes a cyclic prefix of duration $T_{c p}$. Therefore, the duration of each OFDM symbol is $T_{u}=T-T_{c p}$. Every two adjacent subcarriers are spaced by $\delta f=1 / T_{u}$. The output signal of the OFDM system is converted into serial signal by parallel to serial converter. A complex white Gaussian noise process $N\left(0, \sigma_{w}^{2}\right)$ with power spectral density $N_{0} / 2$ is added through a frequency selective time varying multipath fading channel.

In a practical environment, impulse noise can be present, and then the channel becomes nonlinear with non Gaussian impulse noise. The impulse noise can significantly influence the performance of the OFDM communication system for many reasons. First, the time of the arrival of an impulse is unpredictable and shapes of the impulses are not known and they vary considerably. Moreover, impulses usually have very high amplitude, and thus high energy, which can be much greater than the energy of the useful signal [2].

The impulse noise is modeled as a Bernoulli-Gaussian process and it was generated with the Bernoulli-Gaussian process function $i(n)=v(n) \lambda(n)$ where $v(n)$ is a random process with Gaussian distribution and power $\sigma_{B G}^{2}$, and where $\lambda(n)$ is a random process with probability [3]

$P_{r}(\lambda(n))= \begin{cases}p & \lambda=1 \\ 1-p, & \lambda=0 .\end{cases}$

At the receiver, and after removing guard time, the discretetime baseband OFDM signal for the system including impulse noise is

$$
y(n)=\sum_{k=0}^{N-1} X(k) H(k) e^{j \frac{2 \pi}{N} k n}+w(n)+i(n),
$$

$$
=0, \cdots, N-1
$$

where $y(n)$ are time domain samples and $H(k)=$ $D F T_{N}\{h(n)\}$ is the channel's frequency response at the $k^{\text {th }}$ frequency. The sum of both terms of the AWGN noise and impulse noise constitute the total noise given by $z(n)=$ $w(n)+i(n)$.

Let $\Omega_{P}$ the subset of $N_{P}$ pilot subcarriers and $\Delta P$ the pilot interval in frequency domain. Over this subset, channel's frequency response can be estimated, and then interpolated over other subcarriers $\left(N-N_{P}\right)$. These remaining subchannels are interpolated by the nonlinear complex LSSVM algorithm. The OFDM system can be expressed as

$$
\begin{aligned}
y(n)= & y^{P}(n)+y^{D}(n)+z(n) \\
= & \sum_{k \in\left\{\Omega_{P}\right\}} X^{P}(k) H(k) e^{j \frac{2 \pi}{N} k n} \\
& \quad+\sum_{k \notin\left\{\Omega_{P}\right\}} X^{D}(k) H(k) e^{j \frac{2 \pi}{N} k n}
\end{aligned}
$$

where $X^{P}(k)$ and $X^{D}(k)$ are complex pilot and data symbol respectively, transmitted at the $k^{\text {th }}$ subcarrier. Note that, pilot insertion in the subcarriers of every OFDM symbol must satisfy the demand of the sampling theory and uniform distribution [4].

After DFT transformation, $y(n)$ becomes

$$
\begin{array}{r}
Y(k)=D F T_{N}\{y(n)\} \\
=\frac{1}{N} \sum_{n=0}^{N-1} y(n) e^{-j \frac{2 \pi}{N} k n}, \\
=0, \cdots, N-1
\end{array}
$$

Assuming that ISI are eliminated, therefore

$$
\begin{aligned}
Y(k)=X(k) H(k)+W(k) & +I(k)=X(k) H(k)+e(k), \\
& =0, \cdots, N-1
\end{aligned}
$$

where $e(k)$ represents the sum of the AWGN noise $W(k)$ and impulse noise $I(k)$ in the frequency domain, respectively.

Equation (6) may be presented in matrix notation

$$
\begin{aligned}
Y=\boldsymbol{X} \boldsymbol{F} h+W+ & I \\
& =\boldsymbol{X} H+e
\end{aligned}
$$

where

$$
\text { and } \quad W_{N}^{i, k}=
$$

$$
\begin{aligned}
\boldsymbol{X} & =\operatorname{diag}(X(0), X(1), \cdots, X(N-1)) \\
Y & =[Y(0), \cdots, Y(N-1)]^{T} \\
W & =[W(0), \cdots, W(N-1)]^{T} \\
I & =[I(0), \cdots, I(N-1)]^{T} \\
H & =[H(0), \cdots, H(N-1)]^{T} \\
e & =[e(0), \cdots, e(N-1)]^{T}
\end{aligned}
$$$$
\left(\frac{1}{\sqrt{N}}\right) \exp ^{-j 2 \pi\left(\frac{i k}{N}\right)} \text {. }
$$

\section{MULTIPATH CHANNEL MODEL}

We consider the channel impulse response of the frequencyselective fading channel model which can be written a

$$
\begin{gathered}
h(\tau, t)=\sum_{l=0}^{L-1} h_{l}(t) \delta(t \\
\left.-\tau_{l}\right)
\end{gathered}
$$

where $h_{l}(t)$ is the impulse response representing the complex attenuation of the $l^{\text {th }}$ path, $\tau_{l}$ is the random delay of the $l^{\text {th }}$ path and $L$ is the number of multipath replicas. The specification parameters of an extended vehicular A model (EVA) for downlink LTE system with the excess tap delay and the relative power for each path of the channel are shown in table 1 . These parameters are defined by 3 GPP standard [5]. 


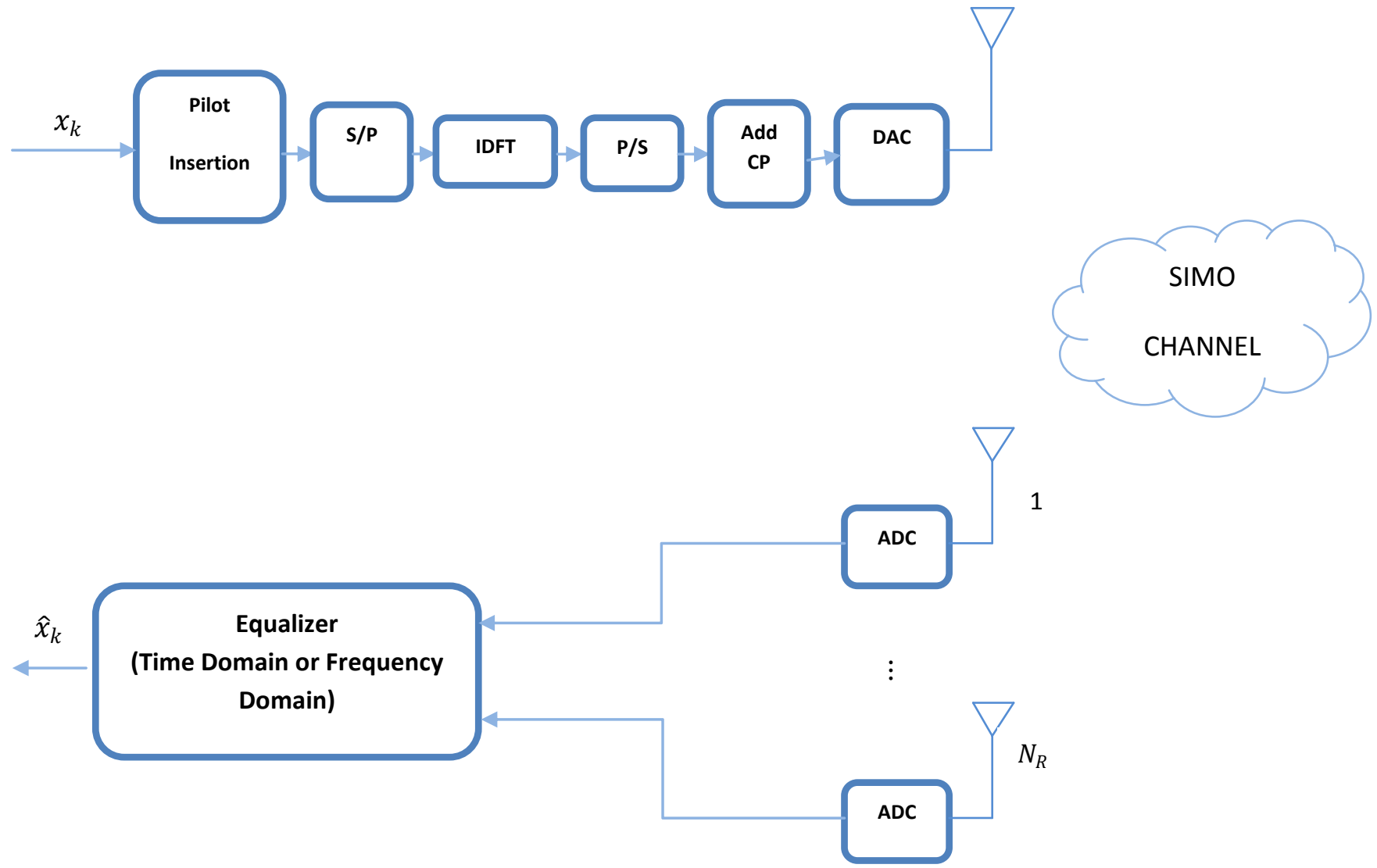

Figure 1. SIMO-OFDM System model

Table 1. Extended Vehicular A model (EVA) [5].

\begin{tabular}{|l|l|}
\hline Excess tap delay [ns] & Relative power [dB] \\
\hline 0 & 0.0 \\
\hline 30 & -1.5 \\
\hline 150 & -1.4 \\
\hline 310 & -3.6 \\
\hline 370 & -0.6 \\
\hline 710 & -9.1 \\
\hline 1090 & -7.0 \\
\hline 1730 & -12.0 \\
\hline 2510 & -16.9 \\
\hline
\end{tabular}

\section{SIMO-OFDM SYSTEM}

Figure (1) shows a SIMO-OFDM system model with $N_{R}$ receive antennas. At the transmitter, the data stream is divided into blocks, of length N. For each block, the inverse IDFT is applied to convert the data into the time domain. Then, a cyclic prefix is added to each block. The time domain blocks are then serially transmitted over a time and frequencyselective multipath fading channel. At the receiver, the $\mathrm{CP}$ is removed and time or frequency domain equalization as well as DFT demodulation is performed to extract the transmitted blocks.
In our considered SIMO system, the diversity receivers extract multiple signal branches of the same signal received from different channels and apply Maximum Ratio Combining (MRC) scheme which gives good performance.

A pilot sequence insertion is used in the channel estimator to predict a refined channel frequency response at the receiver to equalize for the channel impairments and consequently to estimate the transmitted signal.

\section{NONLINEAR COMPLEX LS-SVM ESTIMATOR}

First, note that the index $j$ throughout this section denotes the $\mathrm{j}^{\text {th }}$ antenna at the receiver side of the SIMO system where $j=1, \cdots, N_{R}$.

Let the OFDM frame contains Ns OFDM symbols which every symbol includes $N$ subcarriers. As every OFDM symbol has $N_{P}$ uniformly distributed pilot symbols, the transmitting pilot symbols are $\boldsymbol{X}^{p}=\operatorname{diag}(X(s, m \Delta P)), m=0,1, \cdots, N_{P}-1$, where $s$ and $m$ are labels in time domain and frequency domain respectively.

The proposed channel estimation method is based on nonlinear complex LS-SVM algorithm which has two separate phases: training phase and estimation phase. In training phase, we estimate first the subchannels pilot symbols 
according to LS criterion to strike $\min \left[\left(Y_{j}^{p}-\boldsymbol{X}^{p} \boldsymbol{F} h_{j}\right)\left(Y_{j}^{p}-\right.\right.$ $\left.\left.\boldsymbol{X}^{p} \boldsymbol{F} h_{j}\right)^{H}\right][6]$, as

$$
\begin{aligned}
& \widehat{H}_{j}^{p} \\
= & \boldsymbol{X}^{p-1} Y_{j}^{p}
\end{aligned}
$$

where $Y_{j}^{p}=Y_{j}(s, m \Delta P)$ and $\widehat{H}_{j}^{p}=\widehat{H}_{j}(s, m \Delta P)$ are the received pilot symbols and the estimated frequency responses for the $s^{\text {th }}$ OFDM symbol at pilot positions $m \Delta P$, respectively.

Then, in the estimation phase and by the interpolation mechanism, frequency responses of data subchannels can be determined. Therefore, frequency responses of all the OFDM subcarriers are

$$
\begin{aligned}
& \widehat{H}_{j}(s, q) \\
= & f_{j}\left(\widehat{H}_{j}^{p}(s, m \Delta P)\right)
\end{aligned}
$$

where $q=0, \cdots, N-1$, and $f_{j}(\cdot)$ is the interpolating function, which is determined by the nonlinear complex LS-SVM approach.

In high mobility environments, where the fading channels present very complicated nonlinearities especially in deep fading case, the linear approaches cannot achieve high estimation precision. Therefore, we adapt here a nonlinear complex LS-SVM technique since SVM is superior in solving nonlinear, small samples and high dimensional pattern recognition [4]. Therefore, we map the input vectors to a higher dimensional feature space $\mathcal{H}$ (possibly infinity) by means of nonlinear transformation $\varphi_{j}$. Thus, the regularization term is referred to the regression vector in the RKHS. The following regression function is then

$$
\begin{aligned}
\widehat{H}_{j}(m \Delta P)=\boldsymbol{w}_{j}^{\boldsymbol{T}} \boldsymbol{\varphi}_{j}(m \Delta P)+b_{j} & +e_{j}^{m}, \\
& m=0, \cdots, N_{P}-1
\end{aligned}
$$

where $\boldsymbol{w}_{j}$ is the weight vector, $b_{j}$ is the bias term well known in the SVM literature and residuals $\left\{e_{j}^{m}\right\}$ account for the effect of both approximation errors and noise. In the SVM framework, the optimality criterion is a regularized and constrained version of the regularized LS criterion. In general, SVM algorithms minimize a regularized cost function of the residuals, usually the Vapnik's $\varepsilon$-insensitivity cost function [3]. A robust cost function is introduced to improve the performance of the estimation algorithm which is $\varepsilon$-Huber robust cost function, given by [7]

$$
\begin{aligned}
& \mathcal{L}^{\varepsilon}\left(e_{j}^{m}\right) \\
= & \begin{cases}0, & \left|e_{j}^{m}\right| \leq \varepsilon \\
\frac{1}{2 \gamma}\left(\left|e_{j}^{m}\right|-\varepsilon\right)^{2}, & \varepsilon \leq\left|e_{j}^{m}\right| \leq e_{C} \\
C\left(\left|e_{j}^{m}\right|-\varepsilon\right)-\frac{1}{2} \gamma C^{2}, & e_{C} \leq\left|e_{j}^{m}\right|\end{cases}
\end{aligned}
$$

where $e_{C}=\varepsilon+\gamma C, \varepsilon$ is the insensitive parameter which is positive scalar that represents the insensitivity to a low noise level, parameters $\gamma$ and $C$ control essentially the trade-off between the regularization and the losses, and represent the relevance of the residuals that are in the linear or in the quadratic cost zone, respectively. The cost function is linear for errors above $e_{C}$, and quadratic for errors between $\varepsilon$ and $e_{C}$. Note that, errors lower than $\varepsilon$ are ignored in the $\varepsilon-$ insensitivite zone. On the other hand, the quadratic cost zone uses the $L_{2}-$ norm of errors, which is appropriate for
Gaussian noise, and the linear cost zone limits the effect of sub-Gaussian noise [8]. Therefore, the $\varepsilon$-Huber robust cost function can be adapted to different kinds of noise.

Since $e_{j}^{m}$ is complex, let $\mathcal{L}^{\varepsilon}\left(e_{j}^{m}\right)=\mathcal{L}^{\varepsilon}\left(\mathcal{R}\left(e_{j}^{m}\right)\right)+$ $\mathcal{L}^{\varepsilon}\left(\Im\left(e_{j}^{m}\right)\right)$, where $\mathcal{R}(\cdot)$ and $\mathfrak{I}(\cdot)$ represent real and imaginary parts, respectively.

Now, we can state the primal problem as minimizing

$$
\begin{aligned}
& \frac{1}{2}\left\|\boldsymbol{w}_{j}\right\|^{2}+\frac{1}{2 \gamma} \sum_{m \in I_{1}}\left(\xi_{j}^{m}+\xi_{j}^{m *}\right)^{2}+C \sum_{m \in I_{2}}\left(\xi_{j}^{m}+\xi_{j}^{m *}\right) \\
& +\frac{1}{2 \gamma} \sum_{m \in I_{3}}\left(\zeta_{j}^{m}+\zeta_{j}^{m *}\right)^{2}+C \sum_{m \in I_{4}}\left(\zeta_{j}^{m}+\zeta_{j}^{m *}\right) \\
& -\frac{1}{2} \sum_{m \in I_{2}, I_{4}} \gamma C^{2}
\end{aligned}
$$

constrained to

$$
\begin{gathered}
\mathcal{R}\left(\widehat{H}_{j}(m \Delta P)-\boldsymbol{w}_{j}^{\boldsymbol{T}} \boldsymbol{\varphi}_{j}(m \Delta P)-b_{j}\right) \leq \varepsilon+\xi_{j}^{m} \\
\Im\left(\widehat{H}_{j}(m \Delta P)-\boldsymbol{w}_{j}^{\boldsymbol{T}} \boldsymbol{\varphi}_{j}(m \Delta P)-b_{j}\right) \leq \varepsilon+\zeta_{j}^{m} \\
\mathcal{R}\left(-\widehat{H}_{j}(m \Delta P)+\boldsymbol{w}_{j}^{\boldsymbol{T}} \boldsymbol{\varphi}_{j}(m \Delta P)+b_{j}\right) \leq \varepsilon+\xi_{j}^{m *} \\
\mathfrak{\Im}\left(-\widehat{H}_{j}(m \Delta P)+\boldsymbol{w}_{j}^{\boldsymbol{T}} \boldsymbol{\varphi}_{j}(m \Delta P)+b_{j}\right) \leq \varepsilon+\zeta_{j}^{m^{*}} \\
\xi_{j}^{m{ }^{(*)}}, \zeta_{j}^{m(*)} \\
\geq 0
\end{gathered}
$$

for $m=0, \cdots, N_{P}-1$, where $\xi_{j}^{m}$ and $\xi_{j}^{m *}$ are slack variables which stand for positive and negative errors in the real part, respectively. $\zeta_{j}^{m}$ and $\zeta_{j}^{m *}$ are the errors for the imaginary parts.

$I_{1}, I_{2}, I_{3}$ and $I_{4}$ are the set of samples for which:

$I_{1}$ : real part of the residuals are in the quadratic zone;

$I_{2}$ : real part of the residuals are in the linear zone;

$I_{3}$ : imaginary part of the residuals are in the quadratic zone;

$I_{4}$ : imaginary part of the residuals are in the linear zone.

To transform the minimization of the primal functional (14) subject to constraints in (15), into the optimization of the dual functional, we must first introduce the constraints into the primal functional. Thus, the primal dual functional is as follow:

$$
\begin{aligned}
& L_{P d}=\frac{1}{2}\left\|\boldsymbol{w}_{j}\right\|^{2}+\frac{1}{2 \gamma} \sum_{m \in I_{1}}\left(\xi_{j}^{m}+\xi_{j}^{m *}\right)^{2} \\
& +C \sum_{m \in I_{2}}\left(\xi_{j}^{m}+\xi_{j}^{m *}\right)+\frac{1}{2 \gamma} \sum_{m \in I_{3}}\left(\zeta_{j}^{m}+\zeta_{j}^{m *}\right)^{2} \\
& +C \sum_{m \in I_{4}}\left(\zeta_{j}^{m}+\zeta_{j}^{m *}\right)-\frac{1}{2} \sum_{m \in I_{2}, I_{4}} \gamma C^{2}-\sum_{m=0}^{N_{P}-1}\left(\beta_{j}^{m} \xi_{j}^{m}\right. \\
& \left.+\beta_{j}^{m *} \xi_{j}^{m *}\right)-\sum_{m=0}^{N_{P}-1}\left(\lambda_{j}^{m} \zeta_{j}^{m}+\lambda_{j}^{m}{ }^{*} \zeta_{j}^{m *}\right) \\
& +\sum_{m=0}^{N_{P}-1} \alpha_{\mathcal{R}, m, j}\left[\mathcal{R}\left(\widehat{H}_{j}(m \Delta P)-\boldsymbol{w}_{j}^{T} \boldsymbol{\varphi}_{j}(m \Delta P)-b_{j}\right)-\varepsilon\right. \\
& \left.-\xi_{j}^{m}\right]
\end{aligned}
$$




$$
\begin{gathered}
+\sum_{m=0}^{N_{P}-1} \alpha_{I, m, j}\left[\Im\left(\widehat{H}_{j}(m \Delta P)-\boldsymbol{w}_{j}^{\boldsymbol{T}} \boldsymbol{\varphi}_{j}(m \Delta P)-b_{j}\right)-i \varepsilon\right. \\
\left.-i \zeta_{j}^{m}\right] \\
+\sum_{m=0}^{N_{P}-1} \alpha_{\mathcal{R}, m, j}^{*}\left[\mathcal{R}\left(-\widehat{H}_{j}(m \Delta P)+\boldsymbol{w}_{j}^{\boldsymbol{T}} \boldsymbol{\varphi}_{j}(m \Delta P)+b_{j}\right)-\varepsilon\right. \\
\left.-\xi_{j}^{m}{ }^{*}\right] \\
+\sum_{m=0}^{N_{P}-1} \alpha_{I, m, j}^{*}\left[\Im\left(-\widehat{H}_{j}(m \Delta P)+\boldsymbol{w}_{j}^{\boldsymbol{T}} \boldsymbol{\varphi}_{j}(m \Delta P)+b_{j}\right)-i \varepsilon\right. \\
\left.-i \zeta_{j}^{m}{ }^{*}\right]
\end{gathered}
$$

with the Lagrange multipliers (or dual variables) constrained to $\alpha_{\mathcal{R}, m, j}, \alpha_{I, m, j}, \beta_{j}^{m}, \lambda_{j}^{m} \geq 0, \alpha_{\mathcal{R}, m, j}^{*}, \alpha_{I, m, j}^{*}, \beta_{j}^{m *}, \lambda_{j}^{m *} \geq 0$ and $\xi_{j}^{m}, \zeta_{j}^{m}, \xi_{j}^{m *}, \zeta_{j}^{m *} \geq 0$.

According to Karush-Kuhn-Tucker (KKT) conditions [7]

$\beta_{j}^{m} \xi_{j}^{m}=0, \beta_{j}^{m *} \xi_{j}^{m *}=0$ and $\lambda_{j}^{m} \zeta_{j}^{m}=0, \lambda_{j}^{m *} \zeta_{j}^{m *}=$ 0. (17)

Then, by making zero the primal-dual functional gradient with respect to $\omega_{i j}$, we obtain an optimal solution for the weights

$$
\begin{aligned}
& \boldsymbol{w}_{j}=\sum_{m=0}^{N_{P}-1} \psi_{j}^{m} \boldsymbol{\varphi}_{j}(m \Delta P) \\
&=\sum_{m=0}^{N_{P}-1} \psi_{j}^{m} \boldsymbol{\varphi}_{j}\left(P_{m}\right)
\end{aligned}
$$

where $\psi_{j}^{m}=\left(\alpha_{\mathcal{R}, m, j}-\alpha_{\mathcal{R}, m, j}^{*}\right)+j\left(\alpha_{I, m, j}-\alpha_{I, m, j}^{*}\right) \quad$ with $\alpha_{\mathcal{R}, m, j}, \alpha_{\mathcal{R}, m, j}^{*}, \alpha_{I, m, j}, \alpha_{I, m, j}^{*}$ are the Lagrange multipliers for real and imaginary part of the residuals and $P_{m}=$ $(m \Delta P), m=0, \cdots, N_{P}-1$ are the pilot positions.

We define the Gram matrix as

$$
\begin{array}{r}
\boldsymbol{G}_{\boldsymbol{j}}(u, v)=<\boldsymbol{\varphi}_{j}\left(P_{u}\right), \boldsymbol{\varphi}_{\boldsymbol{j}}\left(P_{v}\right)> \\
=K_{j}\left(P_{u}, P_{v}\right)
\end{array}
$$

where $K_{j}\left(P_{u}, P_{v}\right)$ is a Mercer's kernel which represent in this contribution the RBF kernel matrix which allows obviating the explicit knowledge of the nonlinear mapping $\varphi(\cdot)$. A compact form of the functional problem can be stated in matrix format by placing optimal solution $\boldsymbol{w}_{j}$ into the primal dual functional and grouping terms. Then, the dual problem consists of maximizing

$$
\begin{aligned}
-\frac{1}{2} \boldsymbol{\psi}_{j}^{\boldsymbol{H}}\left(\boldsymbol{G}_{\boldsymbol{j}}+\gamma \boldsymbol{I}\right) & \boldsymbol{\psi}_{\boldsymbol{j}}+\mathcal{R}\left(\boldsymbol{\psi}_{j}^{\boldsymbol{H}} Y_{j}^{p}\right) \\
& -\left(\boldsymbol{\alpha}_{\mathcal{R}, j}+\boldsymbol{\alpha}_{\mathcal{R}, \boldsymbol{j}}^{*}+\boldsymbol{\alpha}_{\boldsymbol{I}, \boldsymbol{j}}\right. \\
+ & \left.\boldsymbol{\alpha}_{\boldsymbol{I}, \boldsymbol{j}}^{*}\right) \mathbf{1} \mathcal{E}
\end{aligned}
$$

Constrained to $0 \leq \alpha_{\mathcal{R}, m, j}, \alpha_{\mathcal{R}, m, j}^{*}, \alpha_{I, m, j}, \alpha_{I, m, j}^{*} \leq C$, where $\boldsymbol{\psi}_{j}^{\boldsymbol{H}}=\left[\psi_{j}^{0}, \cdots, \psi_{j}^{N_{P}-1}\right]^{T} ; \mathbf{I}$ and $\mathbf{1}$ are the identity matrix and the all-ones column vector, respectively; $\boldsymbol{\alpha}_{\mathcal{R}, j}$ is the vector which contains the corresponding dual variables, with the other subsets being similarly represented. The weight vector can be obtained by optimizing (20) with respect to $\alpha_{\mathcal{R}, m, j}, \alpha_{\mathcal{R}, m, j}^{*}, \alpha_{I, m, j}, \alpha_{I, m, j}^{*}$ and then substituting into (18).

Therefore, and after training phase, frequency responses at all subcarriers in each OFDM symbol can be obtained by SVM interpolation

$$
\widehat{H}_{j}(k)=\sum_{m=0}^{N_{P}-1} \psi_{j}^{m} K_{j}\left(P_{m}, k\right)
$$

for $k=1, \cdots, N$. Note that, the obtained subset of Lagrange multipliers which are nonzero will provide with a sparse solution. As usual in the SVM framework, the free parameter of the kernel and the free parameters of the cost function have to be fixed by some a priori knowledge of the problem, or by using some validation set of observations [3].

\section{SIMULATION RESULTS \\ 6.1 SISO CASE}

In order to demonstrate the effectiveness of our proposed technique and evaluate the performance, two objective criteria, the signal-to-noise ratio (SNR) and signal-to-impulse ratio (SIR) are used. The SNR and SIR are given by [3]

$$
\begin{aligned}
& S N R_{d B} \\
= & 10 \log _{10}\left(\frac{E\left\{|y(n)-w(n)-i(n)|^{2}\right\}}{\sigma_{w}^{2}}\right)
\end{aligned}
$$

and

$$
\begin{aligned}
& S I R_{d B} \\
= & 10 \log _{10}\left(\frac{E\left\{|y(n)-w(n)-i(n)|^{2}\right\}}{\sigma_{B G}^{2}}\right)
\end{aligned}
$$

Then, we simulate the SISO-OFDM downlink LTE system with parameters presented in table 2 . The nonlinear complex LS-SVM estimate a number of OFDM symbols in the range of 140 symbols, corresponding to one radio frame LTE. Note that, the LTE radio frame duration is $10 \mathrm{~ms}$ [9], which is divided into 10 subframes. Each subframe is further divided into two slots, each of $0.5 \mathrm{~ms}$ duration.

For the purpose of evaluation the performance of the nonlinear complex LS-SVM algorithm under high mobility conditions, we consider a scenario for downlink LTE system for a mobile speed equal to $350 \mathrm{Km} / \mathrm{h}$. Accordingly, we take into account the impulse noise with $p=.1$ which was added to the reference signals with different rates of SIR.

Table 2. Parameters of simulations [9], [10] and [11].

\begin{tabular}{|l|l|}
\hline Parameters & Specifications \\
\hline Constellation & 16 -QAM \\
\hline Mobile Speed $(\mathrm{Km} / \mathrm{h})$ & 350 \\
\hline$T_{s}(\mu \mathrm{s})$ & 72 \\
\hline$f_{c}(\mathrm{GHz})$ & 2.15 \\
\hline$\delta f(\mathrm{KHz})$ & 15 \\
\hline$B(\mathrm{MHz})$ & 5 \\
\hline Size of DFT/IDFT & 512 \\
\hline Number of paths & 9 \\
\hline
\end{tabular}

Figure (2) presents the variations in time and in frequency of the channel frequency response for the considered scenario. 


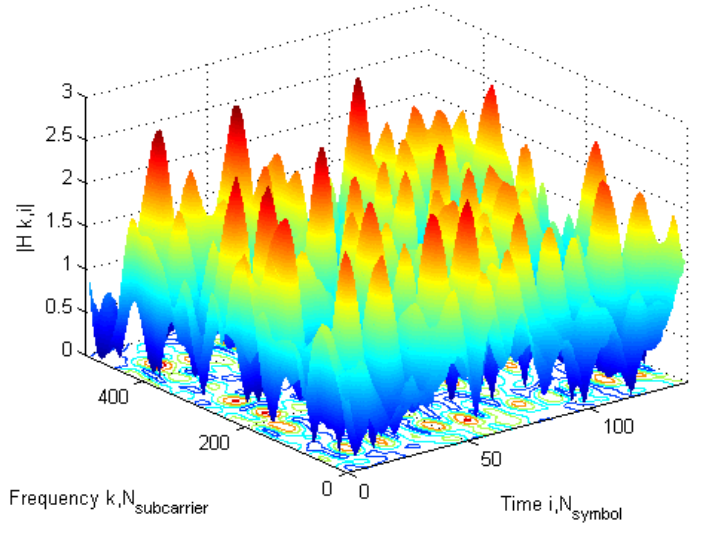

Figure 2. Variations in time and in frequency of the channel frequency response for mobile speed $=350 \mathrm{Km} / \mathrm{h}$.

Figure (3) shows the performance of the LS and nonlinear complex LS-SVM algorithms in the presence of additive Gaussian noise as a function of SNR without impulse noise. A poor performance is noticeably exhibited by LS and better performance is observed with nonlinear complex LS-SVM. These results are confirmed by Figure (4) in the presence of impulse noise for $\mathrm{SIR}=-5 \mathrm{~dB}$ with $p=.1$.

Figure (5) presents a comparison between LS and nonlinear complex LS-SVM in the presence of impulse noise for $\mathrm{SNR}=25 \mathrm{~dB}$ with $p=.1$. The comparison of these methods reveals that nonlinear complex LS-SVM outperforms LS estimator in high mobility conditions especially for high SNR.

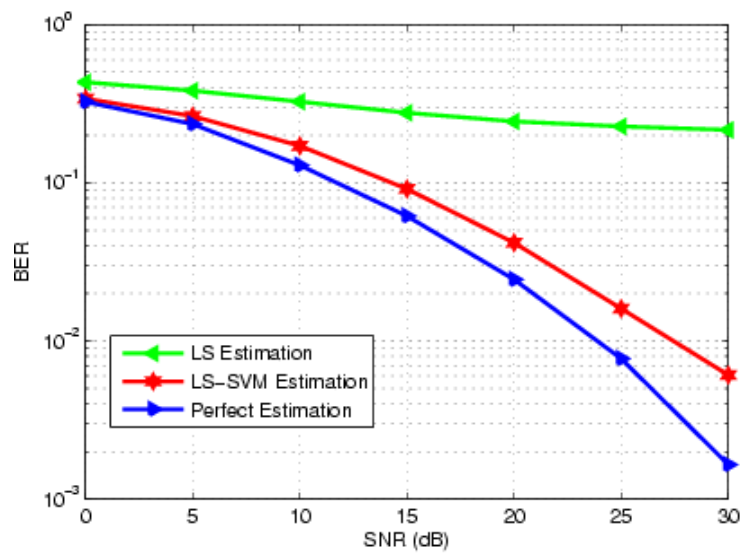

Figure 3. BER as a function of SNR for a mobile speed at $350 \mathrm{Km} / \mathrm{h}$ without impulse noise.

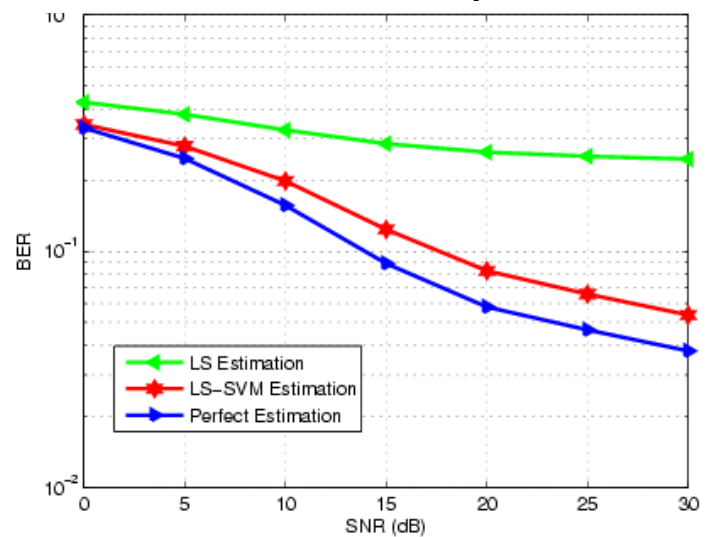

Figure 4. BER as a function of SNR for a mobile speed at $350 \mathrm{Km} / \mathrm{h}$ for $\mathrm{SIR}=-\mathbf{\mathrm { dB }}$ with $\mathrm{p}=.1$.

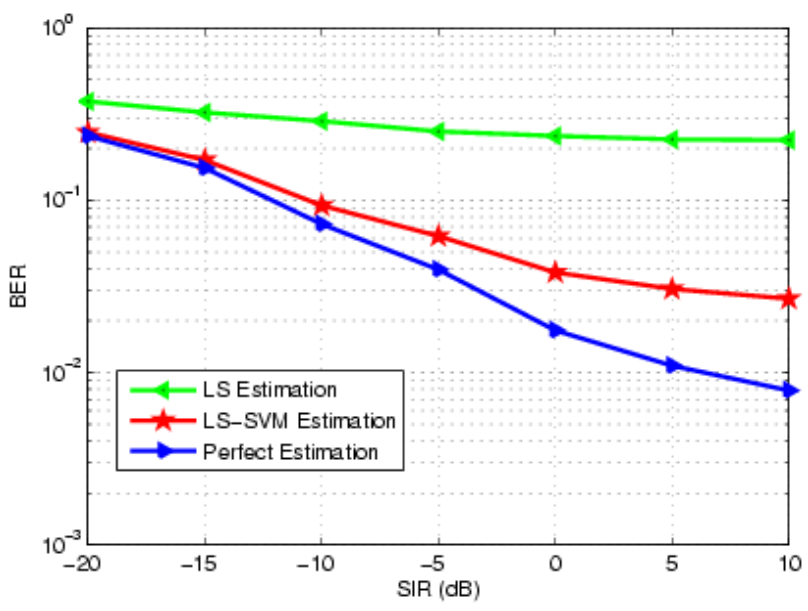

Figure 5. BER as a function of SIR for a mobile speed at $350 \mathrm{Km} / \mathrm{h}$ for $\mathrm{SNR}=25 \mathrm{~dB}$ with $\mathrm{p}=.1$.

\subsection{SIMO CASE}

LTE SIMO-OFDM Downlink system with parameters shown in table 2 is simulated. Also, these parameters are based on Downlink LTE system and the number of receiver antennas. The performance of the proposed estimator is compared to the SISO-OFDM case and evaluated with the variation of the number of receiver antenna.

Figure (6) shows the variations of BER as a function of SNR in the presence of additive Gaussian noise without impulse noise. The proposed method in SIMO case performs better than SISO case, for example at BER $=10^{-3}$ a gain of $15 \mathrm{~dB}$ is obtained. In the presence of impulse noise and for SIR $=-5$ $\mathrm{dB}$ with $p=.1$, the same results are confirmed in Figure (7). We can remark that BER decrease and good performances are obtained when the number of receive antennas increases especially in the scenario of high mobility variations.

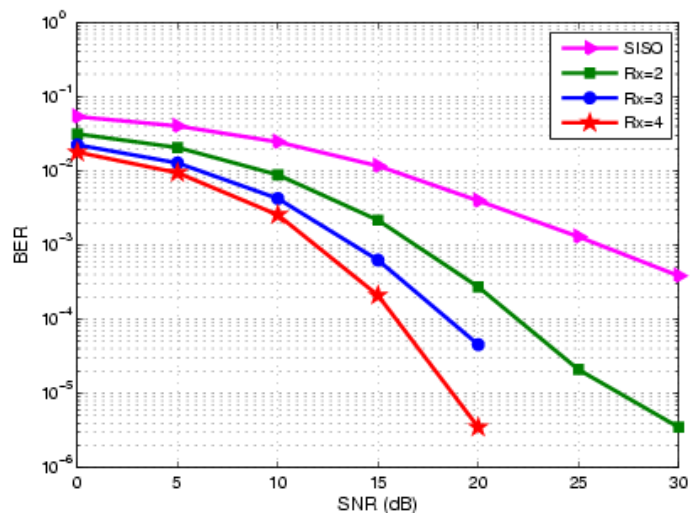

Figure 6. BER as a function of SNR for a mobile speed at $350 \mathrm{Km} / \mathrm{h}$ without impulse noise for the SIMO-OFDM system

Figure (8) presents the performance of the nonlinear complex LS-SVM estimator for the SIMO-OFDM system as a function of SIR in the presence of additive Gaussian noise and impulse noise interfering with pilot signals for a mobile speed at 350 $\mathrm{Km} / \mathrm{h}$ for $\mathrm{SNR}=30 \mathrm{~dB}$ with $p=.1$. The comparison of the nonlinear complex LS-SVM in SIMO and SISO cases reveals that the proposed estimator in the SIMO case outperforms the estimator in SISO case. In fact, good performance is realised 
when the number of receive antennas increases which increases the receive diversity.

\section{CONCLUSION}

In this paper, a new nonlinear complex LS-SVM based channel estimation technique for a highly selective multipath fading downlink SIMO-OFDM LTE system under high mobility conditions in the presence of non-Gaussian impulse noise interfering with OFDM reference symbols is presented.

The proposed channel estimation method is based on learning process that uses training sequence to estimate the channel variations. Our formulation is based on nonlinear complex LS-SVM specifically developed for pilot-based OFDM systems. Simulations have confirmed the capabilities of the proposed nonlinear complex LS-SVM in the presence of Gaussian and impulse noise interfering with the pilot symbols for a high mobile speed when compared to LS standard method. The proposal takes into account the temporal-spectral relationship of the OFDM signal for highly selective channels. The Gram matrix using RBF kernel lead to a significant benefit for OFDM communications especially in those scenarios in which impulse noise and deep fading are presents.

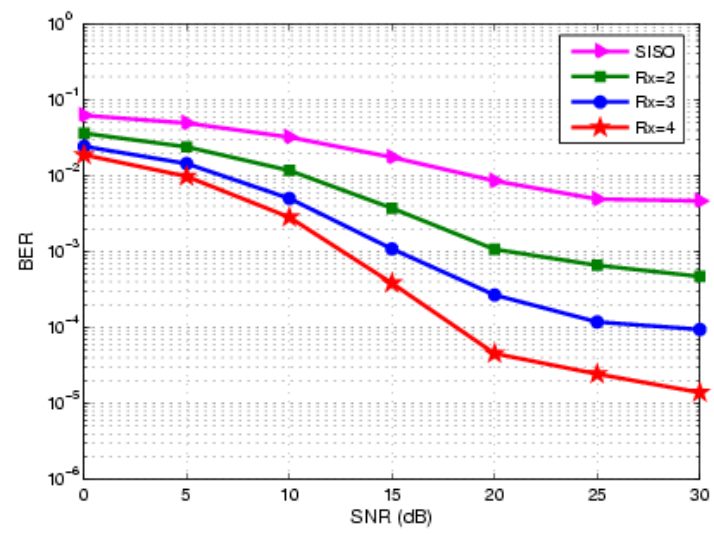

Figure 7. BER as a function of SNR for a mobile speed at $350 \mathrm{Km} / \mathrm{h}$ for $\mathrm{SIR}=-5 \mathrm{~dB}$ with $\mathrm{p}=.1$ for the SIMOOFDM system

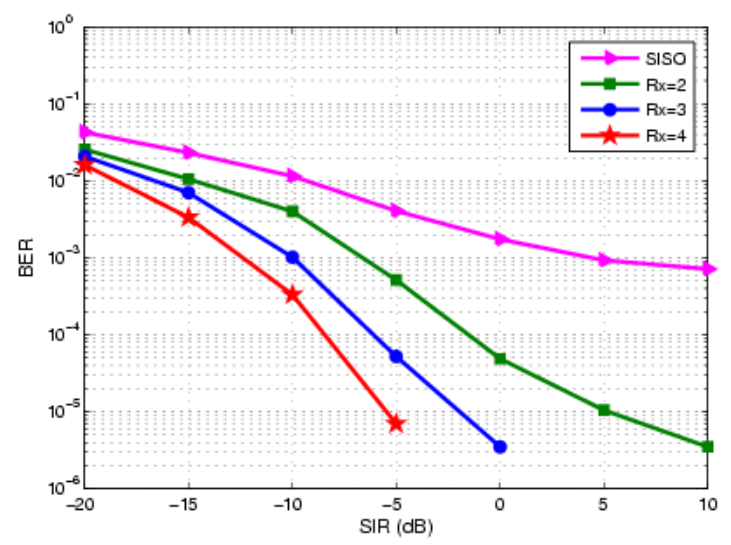

Figure 8. BER as a function of SIR for a mobile speed at $350 \mathrm{Km} / \mathrm{h}$ for $\mathrm{SNR}=30 \mathrm{~dB}$ with $\mathrm{p}=.1$ for the $\mathrm{SIMO}$ OFDM system

\section{REFERENCES}

[1] M. J. Fernández-Getino García, J. M. Páez-Borrallo, and S. Zazo, "DFT-based channel estimation in 2D-pilotsymbol- aided OFDM wireless systems," IEEE Vehicular Technology Conf., vol. 2, pp. 815-819, 2001.

[2] M. Sliskovic, "Signal processing algorithm for OFDM channel with impulse noise," IEEE conf. on Electronics, Circuits and Systems, pp. 222-225, 2000.

[3] J. L. Rojo-Álvarez, C. Figuera-Pozuelo, C. E. MartínezCruz, G. Camps-Valls, F. Alonso-Atienza, M. MartínezRamón, "Nonuniform Interpolation of Noisy Signals Using Support Vector Machines," IEEE Trans. Signal process., vol. 55, no.48, pp. 4116-4126, 2007.

[4] L. Nanping, Y. Yuan, X. Kewen, and Z. Zhiwei, "Study on Channel Estimation Technology in OFDM system," IEEE Computer Society Conf., pp.773-776, 2009.

[5] 3rd Generation Partnership Project, "Technical Specification Group Radio Access Network: evolved Universal Terrestrial Radio Access (UTRA): Base Station (BS) radio transmission and reception," TS 36.104, V8.7.0, September 2009.

[6] S. Coleri, M. Ergen and A. Puri, "Channel estimation techniques based on pilot arrangement in OFDM systems," IEEE Trans. on broadcasting, vol. 48, no.3, pp. 223-229, 2002.

[7] M. Martínez Ramón, N. Xu, and C. G. Christodoulou, "Beamforming Using Support Vector Machines," IEEE antennas and wireless propagation J., vol. 4, 2005.

[8] M. J. Fernández-Getino García, J. L. Rojo-Álvarez, F. Alonso-Atienza, and M. Martínez-Ramón, "Support Vector Machines for Robust Channel Estimation in OFDM," IEEE signal process. J., vol. 13, no. 7, 2006.

[9] 3rd Generation Partnership Project, "Technical Specification Group Radio Access Network: evolved Universal Terrestrial Radio Access (UTRA): Physical Channels and Modulation layer," TS 36.211, V8.8.0, September 2009.

[10] 3rd Generation Partnership Project, “Technical Specification Group Radio Access Network: Physical layer aspects for evolved Universal Terrestrial Radio Access (UTRA)," TR 25.814, V7.1.0, September 2006.

[11] 3rd Generation Partnership Project, "Technical Specification Group Radio Access Network: evolved Universal Terrestrial Radio Access (UTRA): Physical layer procedures," TS 36.213, V8.8.0, September 2009. 